\title{
MULTI-TEMPORAL SAR CHANGE DETECTION AND MONITORING
}

\author{
S. Hachicha, F. Chaabane \\ Carthage University, Sup’Com, COSIM laboratory, Route de Raoued, 3.5 Km, Elghazala Tunisia. \\ ferdaous.chaabene@supcom.rnu.tn
}

\begin{abstract}
KEY WORDS: Spatio-temporal NL-means filtering, Rayleigh Kullback Leibler, Rayleigh Distribution Ratio, DSMT fusion, temporal classification.
\end{abstract}

\begin{abstract}
:
Multitemporal SAR images are a very useful source of information for a large amount of applications, especially for change detection and monitoring. In this paper, a new SAR change detection and monitoring approach is proposed through the analysis of a time series of SAR images covering the same region. The first step of the method is the SAR filtering preprocessing step using an extension of the spatial NL-means filter to the temporal domain. Then, the Rayleigh Kullback Leibler and the Rayleigh Distribution Ratio measures are combined to detect the changes between a reference image and each SAR image of the time series at both local and global scale. These measures are combined using the Dezert-Smarandache theory which takes into account conflicts between sources and thus enhances the dual change detection results. Finally, a pixel based temporal classification is applied starting from the obtained change maps in order to describe the temporal behaviour of the covered regions.
\end{abstract}

\section{INTRODUCTION}

Remote sensing applications have known a fast expansion thanks to the diversity and the amount of satellite images. This paper focuses on the problem of change detection and monitoring. SAR data change detection allows the analysis of land phenomenon for a large range of applications such as the urban and agriculture regions monitoring, the mapping of damages following a natural disaster, etc. These changes can be of different types, origins and durations.

In the literature, several approaches have been proposed for SAR change detection between two images. These methods can be classified in two classes: approaches based on pixel intensity and approaches based on local statistics. The first set of approaches is based on the pixel intensity and depends on the neighbouring pixel intensity inside the analysis window. They include image differencing, log-ratio measures [5], Rayleigh Distribution Ratio (RDR) [3], etc. For the second set of techniques, the local statistics are estimated by considering some reasonable distributions which, in practice, have been found particularly convenient in the case of SAR scenes. Indeed, the first class of measures is limited to the comparison of first order statistics. More information may be extracted from the comparison of the local probability density functions (pdfs).Once the pdfs parameters are estimated, their comparison can also be performed using different criteria and the most usual one is the Kullback-Leibler divergence [4].

All these techniques are designed for comparison between only two SAR images. Not many works were interested to the change detection and monitoring over a time series of SAR images [8]. Most of them are feature and model based Techniques, pixel based clustering techniques or frequent sequential pattern based techniques [6].

In this paper, we are interested in SAR change detection and monitoring through the analysis of a time series of SAR images covering the same region [10]. The aim of this work is to consider the radiometric information and to characterize the change by its temporal signature and evolution. Then the investigation concerns the identification of the changes and their monitoring using a temporal classification. We believe that changes can be categorized in four classes:

- Abrupt changes such as urban constructions, deforestation area, etc,

- Evolutionary changes such as vegetation areas,

- No changes areas i.e. stable areas as urban areas for example,

- Periodic changes such as the vegetation evolution according to seasons.

Thus, dual SAR change detection indicators are going to be used in order to classify the changes according to their temporal behavior.

The main contribution of this work consists in the introduction of a judicious combination of two families SAR change detection indicators in the change monitoring approach which can be considered as an improvement of the work done in [10]. Indeed, the proposed technique is based on a combination between a local statistics and a pixel intensity change detection indicators. It suggests an original strategy for changes temporal classification using the behaviour of the dual change measures between each SAR image and a constructed reference image. The proposed multi-temporal change detection and monitoring approach follows these main steps (cf. figure1):

a) As a preprocessing step, we apply a spatio-temporal adaptive filtering of the SAR sequence to reduce the speckle noise and estimate the reflectivity [10].

b) Construction of the reference image which represents an intermediate state throughout the SAR time series.

c) Identification of the changes between the generated reference image and each filtered SAR image using a local statistics change detection family operator which assumes a Rayleigh distribution for a non local neighbourhood [10]. The contribution of this paper is to introduce a pixel intensity change detection indicator applied on non filtered images. Thus, the local statistics and a pixel intensity change detection measures are combined using the DSM theory [11] in order to detect temporal changes. Both local and global variations are then detected by the resulted detector. 


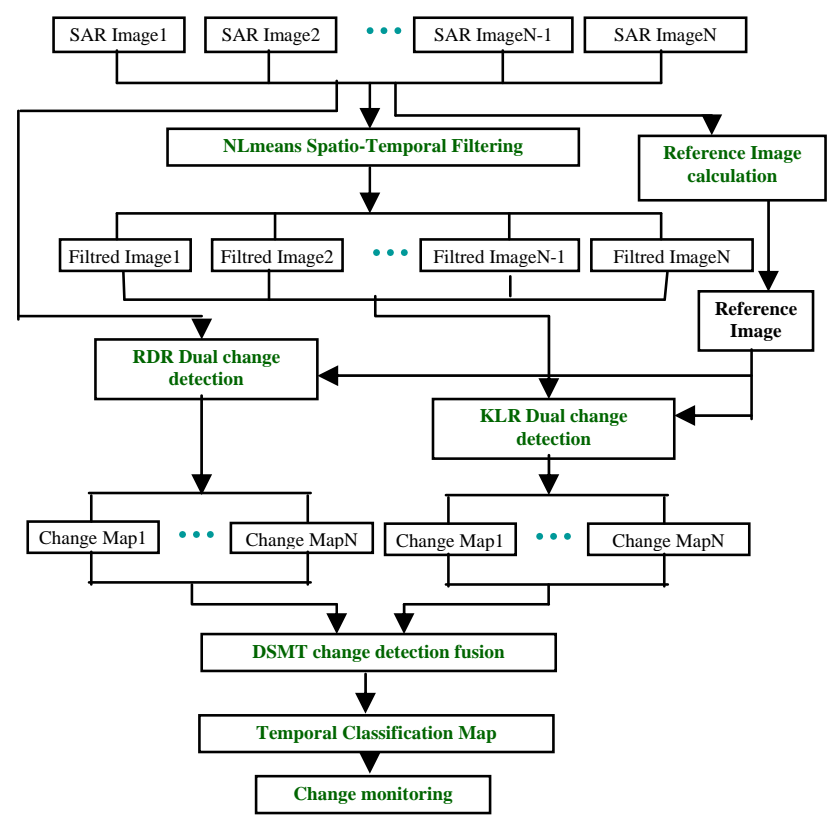

Figure 1. SAR Change detection and monitoring bloc diagram.

\section{NL MEANS SPATIO-TEMPORAL FILTERING}

As SAR images are inherently affected by the speckle which can be described as a multiplicative noise, the first step concerns a spatial and temporal adaptive filter which aims to reduce the speckle noise and to maximize the discrimination capability between the unchanged and the changed classes. The considered filter is based on the same scheme as the Non-local means filter [1] by substituting the Euclidean distance with a similarity criterion adapted to speckle noise which is the Rayleigh Distribution Ratio (RDR).

The weights [2], depending on the RDR indicator, are based on the similarity between a noisy patch that surrounds the central pixel and the ones that surround a given neighbouring pixel in the spatial and the temporal domain. The filtered reflectivity is the given by this expression [10]:

$$
\hat{R}_{f}=\frac{\sum_{t_{x}^{\prime}=1}^{N} \omega\left(t, t_{x}^{\prime}\right) A_{t_{x}^{\prime}}^{2}}{\sum_{t_{x}^{\prime}=1}^{N} \omega\left(t, t_{x}^{\prime}\right)}
$$

where $\omega\left(t, t_{x}{ }^{\prime}\right)$ defines the similarity between patches from $t$ to $t_{x}{ }^{\prime}$ images, $A_{t^{\prime} x}$ is the SAR amplitude and $N$ is the number of images in the temporal sequence series.

This non-local patch-based filter leads to define a filtered image for each SAR image of the time series. The filtered and non filtered images are then compared to the reference image according to suitable change detectors combination in a next step.

\section{REFERENCE IMAGE CALCULATION}

The second step of the proposed approach concerns the reference image calculation. The reference image corresponds to the most stable state of the spatio-temporal patches for a given pixel through the Non-local means filter applied in the first step. That's why it is considered as an intermediate image throughout the time sequence series and the temporal changes will be considered according to this stable state.
For the time series SAR images, we calculate this intermediate image by extracting, for each pixel, the maximum number of the most similar spatio-temporal patches using the NL means weights already calculated in the first step.

The reference image is then calculated starting from the original SAR images. However, since the change detector is applied on filtered SAR images, the reference image is also spatially filtered using the spatial NL means filter.

\section{CHANGE DETECTION MEASURES}

In this paper, we take into account the radar signal features. SAR images are inherently affected by the speckle which can be described as a multiplicative noise. Indeed, if the intensity $I$ follows an exponential law with parameter $R$, we can write $I$ as:

$I=R S$

where $s$ defines a random variable following an exponential distribution with parameter 1 . The same explanation can be done with the SAR amplitude by considering a multiplicative factor $R$. Thus, despeckling a radar image comes back to suppress the factor $s$ in the equation 2, which is equivalent to estimate the reflectivity $R$ in each pixel of the image. Therefore, filtering radar image intensity will be obtained directly by estimating the parameter $R$, and despeckling radar image amplitude will be obtained by considering $\sqrt{R}$.

\subsection{Rayleigh Kullback-Leibler measure}

Since the adaptive spatio-temporal NL means filtering (section 2) reduces considerably the speckle noise without resolution loss, the estimated reflectivity $\hat{R}_{f}$ should describe as close as possible the Rayleigh distribution of noisy data. Thus, a Rayleigh Kullback-Leibler [3] (RKL) based measure is applied in order to detect changes between the generated filtered reference image and each filtered SAR image.

The RKL measure belongs to the local statistics change detection family operators and assumes a Rayleigh distribution. This symmetric Kullback-Leibler divergence measure is given by:

$$
K L_{\text {Rayleigh }}(X, Y)=L \frac{\left|\hat{R}_{f_{X}}-\hat{R}_{f_{Y}}\right|^{2}}{\hat{R}_{f_{X}} \hat{R}_{f_{Y}}}
$$

where $\mathrm{X}$ and $\mathrm{Y}$ designate, for a given pixel, the filtered reference image and the taken filtered SAR image.

\subsection{RDR measure}

Besides, since the SAR amplitude follows a Rayleigh distribution and by using the logarithm of noisy amplitudes ratio which can transform the multiplicative speckle noise into additive, we apply the Rayleigh Distribution Ratio (RDR) used for filtering process as a change detection measure [3]. This indicator is given by:

$$
R D R\left(p, p^{\prime}\right)=\frac{1}{N} \sum_{(k, l) \in V} \log \left(\frac{A_{X_{p}}(k, l)}{A_{Y_{p^{\prime}}}(k, l)}+\frac{A_{Y_{p^{\prime}}}(k, l)}{A_{X_{p}}(k, l)}\right)
$$

where $V$ defines a neighbourhood for the pixel $p$ or $p$ ' which is a window of size $w$. The RDR operator is then applied on non filtered amplitude SAR images. 


\subsection{Change detection measures fusion}

Then the RKL based measure and the RDR measures are combined using the Dezert-Smarandache Theory [11]. The DSMT takes into account conflicts between sources which enhance dual change detection results (between the generated reference image and each SAR image), thus multitemporal change detection results. Both local and global variations are then considered by the resulted detector.

\section{CHANGE DETECTION AND MONITORING}

The last step concerns the temporal changes classification which is not straightforward, since time series contain a combination of seasonal, gradual and abrupt changes. Thus, we propose a generic pixel change classification approach for time series change images based on conventional K-means classification algorithm. This step allows the generation of a temporal map describing the spatial areas according to their temporal behaviour which can be very interesting in many applications.
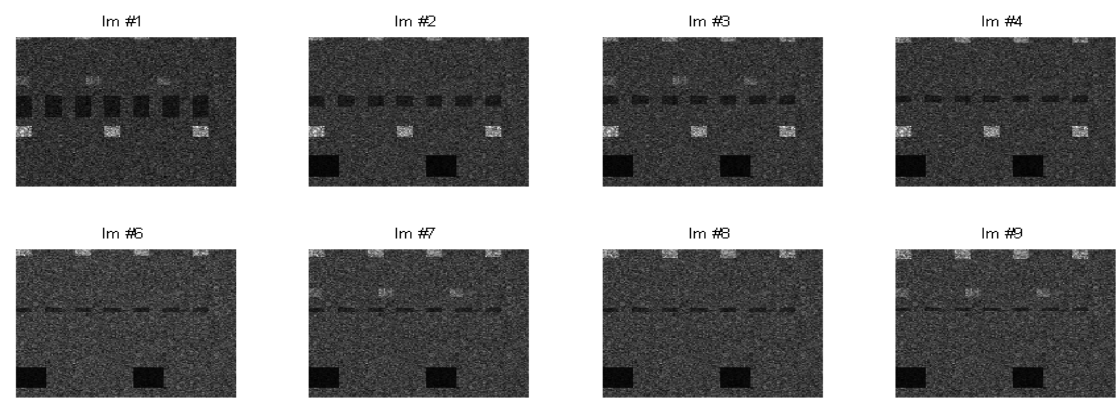

Im \#11
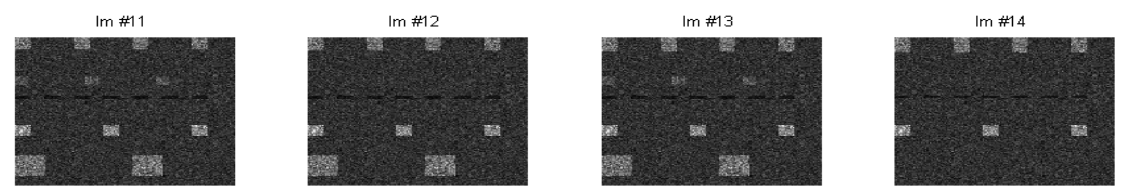

Figure 2. Simulated SAR images time series (15 images).
In this section, we will validate the effectiveness of the proposed approach on both simulated and real datasets. It is first validated on simulated SAR images then applied on real images.

\subsection{Case of simulated data}

The proposed approach was applied first on synthetic data which simulate most of the categories of the temporal changes and then validated in terms of Overall Accuracy (OA) coefficient. representing most of the temporal changes categories: stable (the background), growing, abrupt appearance and periodic areas. As we can see most of the regions have a different temporal behaviour.
The filtering results (as presented in Figure 3) demonstrate a great reduction of the speckle noise and improve the changes discrimination as proved in [10].
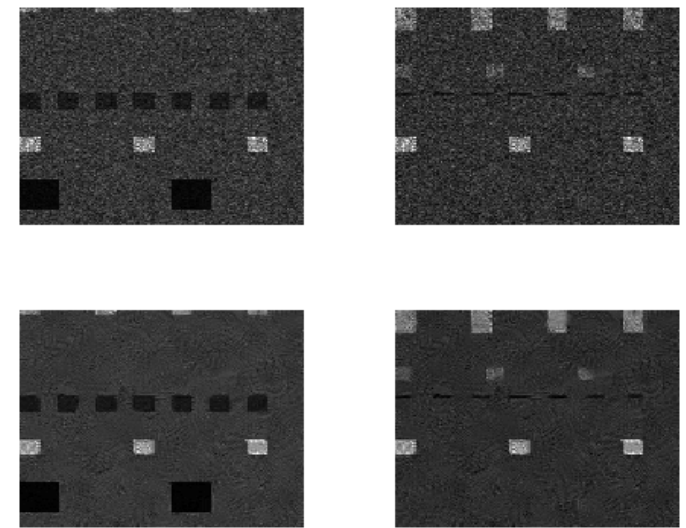

Figure 3. Examples of simulated images filtered by the proposed Spatio-Temporal NL-means filter.

Figure 4 shows the resulted reference image which corresponds to an intermediate state of the simulated images since it is generated starting from the most regular and stable patches.
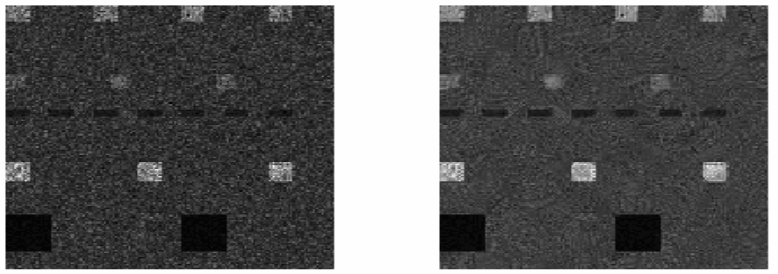

Figure 4. The resulted non filtered and filtered Reference image.

Figure 5 presents the change maps obtained using the RKL, the RDR measures and the DSMT fusion. As can be seen from this Figure, the fusion change maps illustrate a better delimitation of the changing areas. 

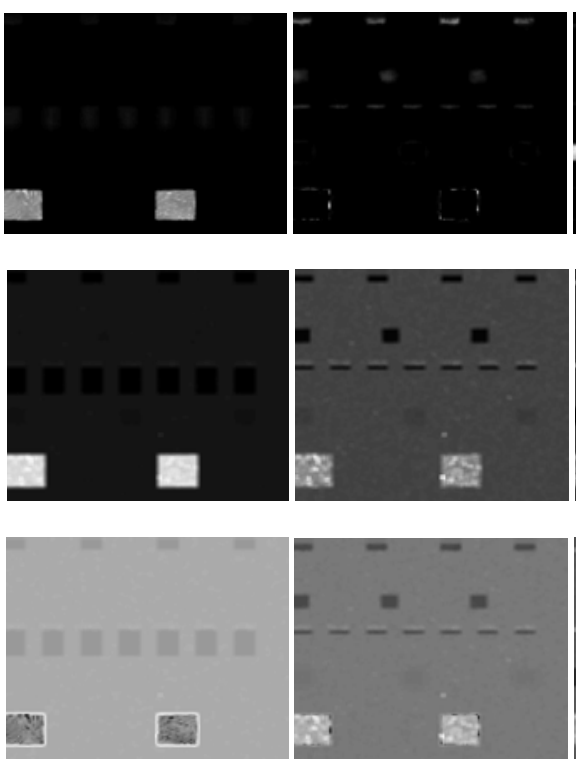

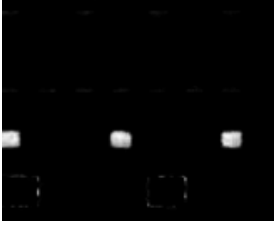

(a)

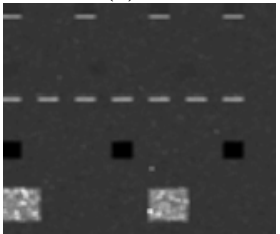

(b)

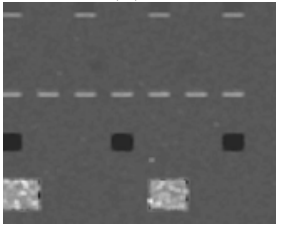

(c)
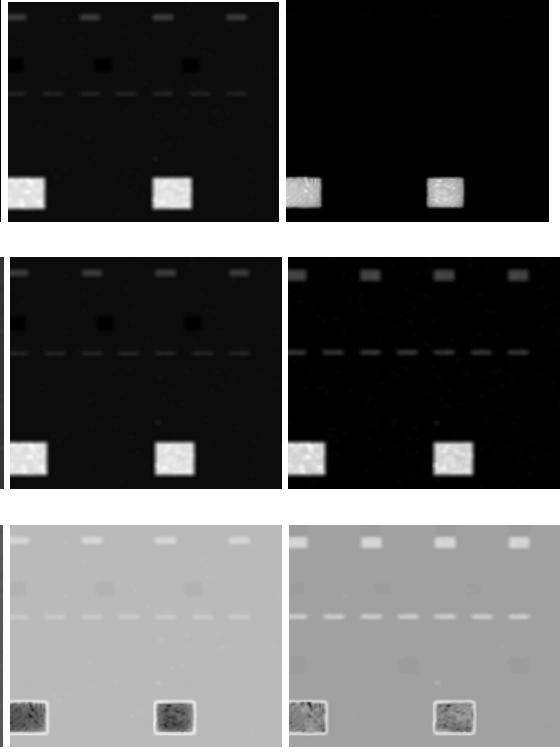

霡

Figure 5. Change detection maps (a) RKL change maps (b) RDR change maps (c) DSMT fusion results.

Figure 6 illustrates the temporal classification of the change detection maps obtained by using the K-means algorithm. As we can see the proposed approach puts in evidence all the change classes' categories which have been simulated.

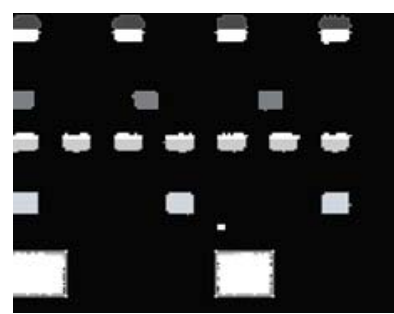

(a)

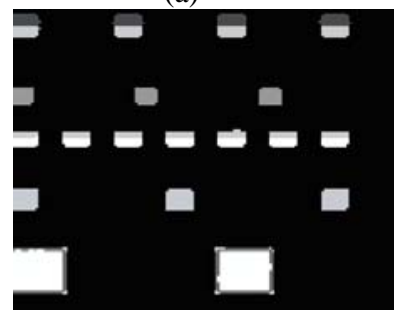

(c)

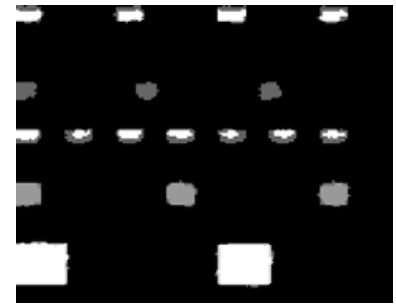

(b)

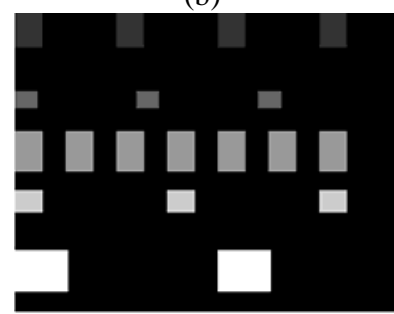

(d)
Legend: $\square$ Stable areas $\square \square$ Abrupt changes

$\square$ Periodic behaviour $\square$ Evolutionary behaviour.

Figure 6. Temporal Classification results. (a) RDR temporal classification results, $\mathrm{OA}=0.67$, (b) $\mathrm{RKL}$ temporal classification results, $\mathrm{OA}=0.61$, (c) DSMT fusion temporal classification results, $\mathrm{OA}=0.72$, (d)

Temporal ground truth image.

\subsection{Case of real data}

Then, the proposed approach is applied on a set of 14 ENVISAT SAR images covering the region of Tunis City (cf. Figure 7) which is a developing region touched by several kinds of changes. As we can notice, The 14 SAR images cover a large temporal period.

Figure 8 corresponds to the obtained and filtered reference image. As we can see, it matches the most stable areas according to original images (cf. figure 7). We can also notice that the NL-means filtering preserve the SAR information.

Figure 9 shows some DSMT based change detection maps generated throughout the proposed approach. As we can see some changed regions are highlighted by the detection process. These maps are then used to generate the temporal classification map which is illustrated by figure 10 .

As can be noticed from this figure, most of the stable areas are correctly delimited. The black region at the right down side which has a periodic behaviour (see figure 7) is also rightly classified.
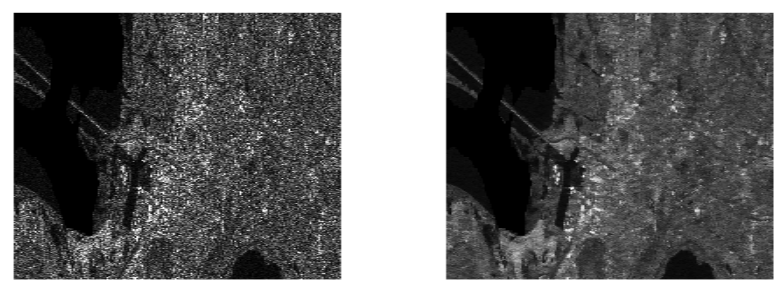

Figure 8 . The resulted non filtered and filtered Reference image for real data. 
Im \#1

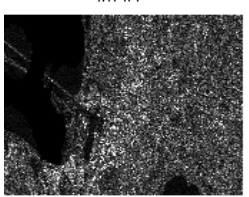

Im

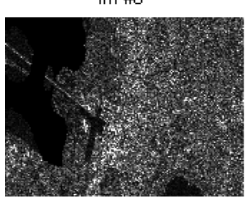

Im $\# 1$

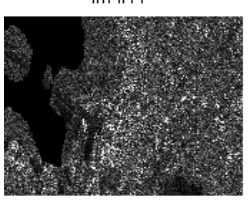

Im \#2

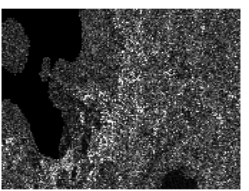

Im \#

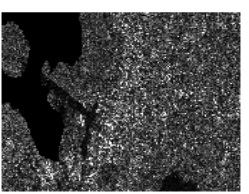

Im \#12

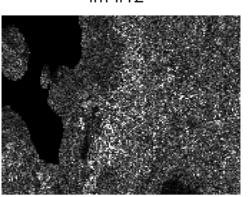

Im \#3

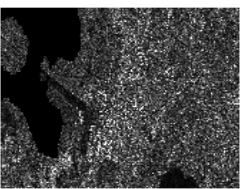

Im $\#$

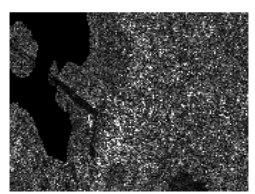

Im \#13

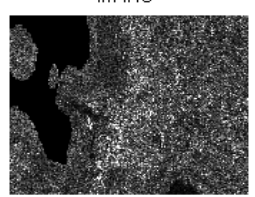

Im \#4

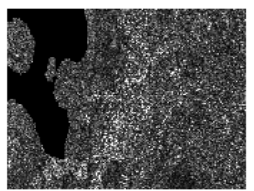

Im

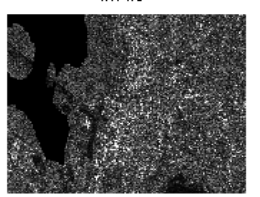

Im \#14

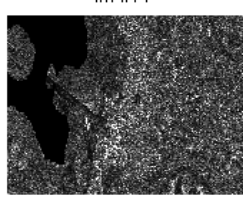

Im 却

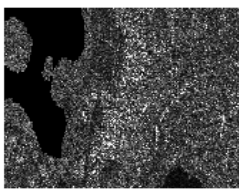

Im \#10

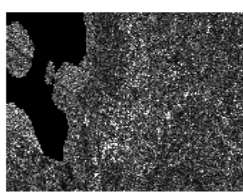

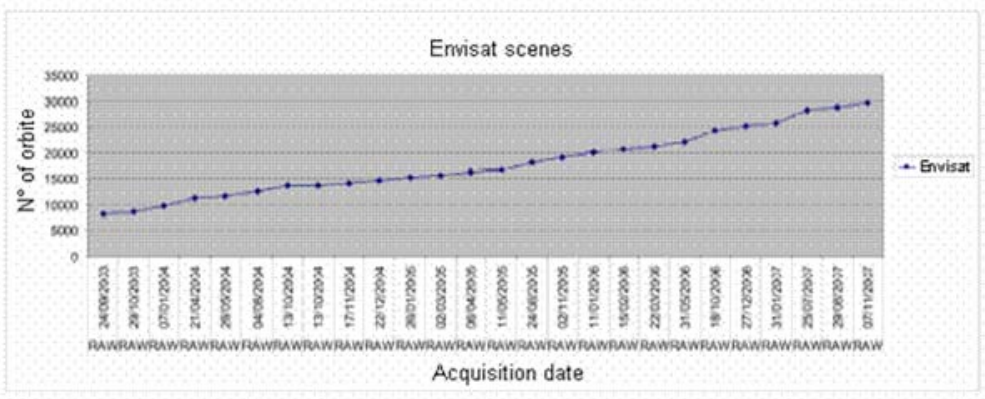

Figure 7. Original SAR Images time series and the corresponding temporal covering.
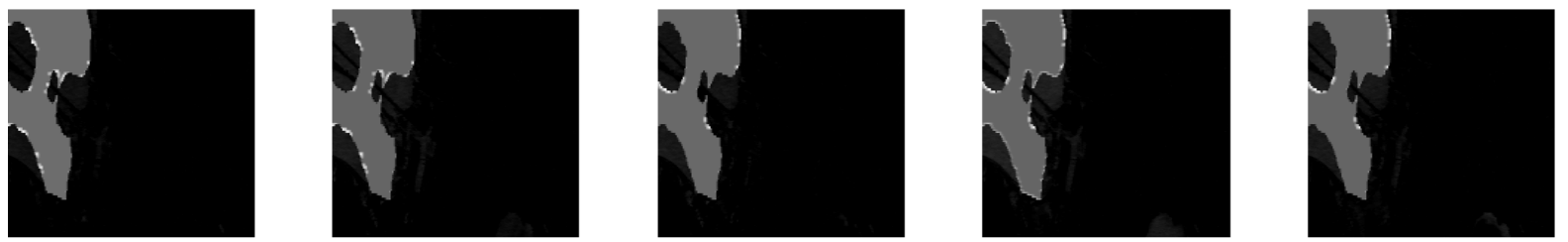

Figure 9. Examples of DSMT change detection maps selected through the SAR images time series.

Legend: $\square$ Periodic behaviour $\square$ Stable areas $\square$ Evolutionary behaviour $\square$ Abrupt changes.

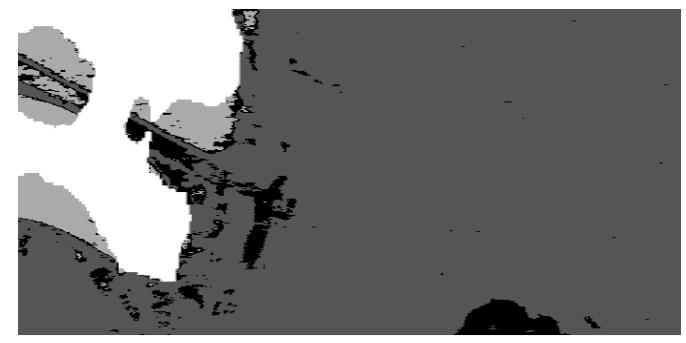

Figure 10. Real data DSMT temporal Classification results.

\section{CONCLUSION}

We have proposed in this paper a change detection and monitoring approach based on combination between two categories of change measures. As the Rayleigh KL and RDR measures take into account the radar signal features, they are applied to estimate the temporal changes. Those measures are combined to take into account complementarities and similitudes between sources which enhance change detection results, thus the temporal classification results.

The proposed approach has been validated on both simulated and real data and we obtain very satisfactory results. 


\section{REFERENCES}

[1] A. Buades, B. Coll, and J. Morel, 2005. A Non-Local Algorithm for Image Denoising. Computer Vision and Pattern Recognition, 2005.CVPR 2005.IEEE Computer Society Conference on, Vol. 2.

[2] C. Deledalle, L. Denis, and F. Tupin, 2009. Iterative Weighted Maximum Likelihood Denoising with Probabilistic Patch-Based Weights”, IEEE Transactions on Image Processing.

[3] S. Hachicha and F. Chaabane 2010. Comparison of change detection indicators in SAR images. EUSAR, pp. 109-112.

[4] J. Inglada and G. Mercier, 2007. A New Statistical Similarity Measure for Change Detection in Multitemporal SAR Images and Its Extension to Multiscale-Change Analysis. IEEE TGRS, 45(5), pp 1432-1446.

[5] R. Touzi, A. Lopes, and P. Bousquet, 1988. A statistical and geometrical edge detector for SAR image. IEEE TGRS, 26(6), pp. 764-773.

[6] A. Julea, N. Méger, P. Bolon, C. Rigotti, M.P. Doin, C. Lasserre, E. Trouvé, V. N. Lazarescu, 2011. Unsupervised Spatiotemporal Mining of Satellite Image Time Series Using Grouped Frequent Sequential Patterns. IEEE TGRS. 49(4), pp. 1417-1430.

[7] I. Bloch, 1996. Information Combination Operators for Data Fusion: A Comparative Review with Classification. IEEE Systems, Man and Cybernetics. 26(1), pp. 52-67.

[8] F. Bujor, E. Trouve, L. Valet, J.-M. Nicolas, and J.-P. Rudant, 2004. Application of log-cumulants to the detection of spatiotemporal discontinuities in multitemporal sar images. IEEE Transactions on Geoscience and Remote Sensing, pp. 2073-2084.

[9] F. Chaabane, A. Avallone, F. Tupin, P. Briole and H. Maître, 2007. A Multitemporal Method for Correction of Tropospheric Effects in Differential SAR Interferometry: Application to the Gulf of Corinth Earthquake," in IEEE Transactions on Geoscience and Remote Sensing, pp. 16051615.

[10] S. Hachicha, C. Deledalle, F. Chaabane and F. Tupin, 2011. Multi-temporal SAR classification according to change detection operators, The IEEE 6th International Workshop on the Analysis of Multi-Temporal Remote Sensing Images.

[11] S. Hachicha and F. Chaabane, 2009. Application of DSm Theory for SAR image change detection. IEEE International Conference on Image Processing. 